\title{
Devenir agricultrice : à la frontière de la vie domestique et de la profession
}

Becoming a woman farmer: at the junction of household activities with agricultural profession

\section{Alice Barthez}

\section{(2) OpenEdition}

\section{Journals}

Édition électronique

URL : http://journals.openedition.org/economierurale/102

DOI : 10.4000/economierurale.102

ISSN : 2105-2581

\section{Éditeur}

Société Française d'Économie Rurale (SFER)

Édition imprimée

Date de publication : 30 décembre 2005

Pagination : 30-43

ISSN : 0013-0559

Référence électronique

Alice Barthez, «Devenir agricultrice : à la frontière de la vie domestique et de la profession », Économie rurale [En ligne], 289-290 | Septembre-décembre 2005, mis en ligne le 02 mars 2009, consulté le 19 avril 2019. URL : http://journals.openedition.org/economierurale/102 ; DOI : 10.4000/ economierurale.102

Ce document a été généré automatiquement le 19 avril 2019.

(c) Tous droits réservés 


\title{
Devenir agricultrice : à la frontière de la vie domestique et de la profession
}

\author{
Becoming a woman farmer: at the junction of household activities with \\ agricultural profession
}

\section{Alice Barthez}

1 S'interroger sur le travail des agricultrices soulève inlassablement la question de la légitimité du travail féminin et de sa conciliation avec l'activité domestique considérée comme le pôle principal de la compétence «naturelle » des femmes. La notion de «travail productif » est, dès l'origine, « un construit sexué » (Daune-Richard, 2002). Quand au XIX ${ }^{\mathrm{e}}$ siècle surgit la notion de "population active », la statistique nationale trace la frontière qui sépare l'activité de «l'inactivité » (Fouquet, 2004) et les femmes se trouvent instituées du mauvais côté. Jusqu'au début du siècle dernier, l'activité productive a massivement lieu dans des unités familiales. Dans la mesure où la profession s'exerce dans la famille, les femmes participent à l'activité productive mais seul le chef de famille est désigné comme chef d'entreprise, tandis que les autres membres de la famille sont classés comme « dépendants ».

2 Les femmes ont toujours travaillé (Schweitzer, 2002) mais leur participation à la production marchande, quand elle s'exerce dans la famille, reste invisible. Depuis le Code Napoléon (1804), les femmes sont définies comme mineures. Mariées, elles se trouvent dépendantes de leur mari pour tout acte concernant la gestion de leurs biens propres et ne peuvent exercer une profession que sous le contrôle de celui-ci. La montée massive du salariat ainsi que de profonds changements dans l'institution de la famille, modifient la place des femmes dans la société.

3 L'agriculture fait partie des Indépendants et «l'exploitation familiale », forme l'archétype de la négation du travail des femmes (Barthez, 1982). La solution la plus directe a été pour elles, l'exode mais elle n'est pas la seule. Aujourd'hui, la population active agricole compte un tiers de femmes. 
Cet article propose de montrer comment celles qui participent à la production agricole sont parvenues peu à peu à faire reconnaître leur activité dans une unité qui ne distingue toujours pas l'univers professionnel de la famille. Toutefois, l'introduction des critères de la production marchande dans les relations de parenté bousculent l'opposition communément admise entre solidarité familiale et intérêt professionnel, entre l'amour et l'argent (Gramain et Weber, 2003).

5 La généralisation du salariat, les transformations de la famille et les modifications en profondeur de l'activité agricole ont formé un cadre favorable à une évolution de la place des femmes en agriculture. Outre les travaux de sociologues, seront utilisées les études de la statistique sur le travail féminin faisant de la reconnaissance professionnelle des femmes une question toujours d'actualité. Enfin, la presse agricole, les journaux syndicaux, les publications de divers organismes professionnels, des livres témoignages, récits de vie, ont été ici mobilisés en tant qu'ils participent à l'élaboration du portrait social de l'agricultrice.

\section{La femme de la campagne " pièce rapportée ", aide familiale...}

6 Jusque dans les années 1960, les femmes, qui participent à la mise en valeur de la ferme familiale, sont considérées par leur place dans la famille et non par l'importance du travail qu'elles réalisent. Dans cette logique, les femmes ne «travaillent» pas, elles « aident » leur mari. "Aide familiale » est leur désignation par rapport à leur conjoint, «chef d'exploitation». Selon leur définition administrative, elles sont établies «sans profession » ce qui les présuppose disponibles au gré des besoins des autres membres de la famille dans leur vie domestique mais aussi dans leur activité productive. Dans le sud de la France, l'existence de la cohabitation entre générations en référence au modèle de la «famille souche » décrite au XIX siècle par Frédéric Le Play (Kalaora et Savoye, 1989), place la femme sous la dépendance non seulement de son mari mais aussi de sa lignée. La belle-fille soumise à l'autorité de sa belle-mère est avant tout une " pièce rapportée » à un ensemble préexistant dont elle doit adopter les règles et participer à son développement par sa capacité procréatrice et par son travail dans ce qui forme le patrimoine de sa bellefamille.

7 L'organisation du travail en agriculture implique une répartition de l'autorité, une division des tâches selon des critères empruntés à la vie familiale et non à la profession. L'aptitude à exercer l'activité agricole est liée à des différences biologiques d'âge, de sexe et de place de chacun dans la parenté. Les historiens ont montré combien les travaux sont sexués et les espaces délimités. Ainsi, au début du siècle dernier : "Semer et labourer, moissonner, faucher, charger et conduire les charrettes, panser les chevaux, nettoyer l'écurie, abattre les arbres sont à l'évidence des travaux d'homme; la cuisine, l'entretien du linge, l'éducation des jeunes enfants, les soins à la basse-cour, des travaux de femme.» (Duby, 1976). Les travaux d'ethnographie décrivent comment dans l'ancienne France, hommes et femmes se répartissent l'espace villageois, de manière à former une complémentarité objective entre les sexes: "Les hommes sont aux champs, à la forge, mais aussi au café, au terrain de jeu communal. Les femmes sont au lavoir, à la fontaine, au four communal, dans la boutique d'épicerie. C'est le prolongement extérieur de leurs tâches domestiques qui mène les 
femmes hors de chez elles. Elles sortent avec une motivation de nature ménagère.» (Ségalen, 1980).

8 Le travail des femmes ne se limite pas toujours à l'espace de la maison et de la cour de ferme même si on ne leur attribue que celui-là. Il s'étend à l'ensemble de l'activité agricole. Elles participent aux travaux des champs notamment à des moments cruciaux tels que les moissons, les semailles ou l'entretien de parcelles cultivées. Dans les régions d'élevage, elles travaillent à l'étable, aux soins des animaux et à la traite des vaches laitières (Bécouarn, 1970). Mais quoiqu'elles fassent, seul l'homme est reconnu comme chef d'exploitation détenteur du pouvoir de décision sur la vie de l'entreprise et son orientation. Pourtant, à un moment de leur trajectoire hommes et femmes ont le même statut : les enfants (filles et garçons) participent à la mise en valeur de la ferme comme leur mère, en tant qu'aides familiaux. Mais cela ne dure qu'un temps : le fils se destine à devenir chef d'exploitation tandis que la fille vise le mariage comme la seule voie d'insertion sociale pour elle. Rester célibataire est un échec, la "vieille fille", marginalisée. Pourtant le mariage désigne une position d'infériorité qui empêche la femme d'être reconnue pour elle-même. Prise dans le réseau de parenté de son mari, elle est appelée à se soumettre à un ensemble de règles qui la placent au service des autres. Quand à cela s'ajoute la cohabitation entre générations, la femme est privée d'autonomie au sein du ménage. La vie de couple est impossible, seule la maisonnée s'impose avec son organisation spécifique.

9 À partir des années 1960, les épouses ainsi instituées en « pièce rapportée » parviennent à rompre le silence et à mettre des mots sur leur situation dominée : «Je ne me sens heureuse qu'au milieu des champs. La vie d'intérieur me répugne car je dois supporter la présence de ma belle-mère qui régente tout.» (Allauzen, 1967) ${ }^{1}$. Le poids des rapports entre générations ne s'exerce pas uniquement sur les femmes. Les jeunes épouses dénoncent le rapport patriarcal qui pèse sur leur mari : "Mon beau-père est très autoritaire. Il nous critique devant les enfants et prend leur parti contre nous. Il a beaucoup d'influence sur mon mari qui le craint et lui obéit toujours, comme avant notre mariage.»

En agriculture, la modernisation des techniques de production, l'introduction d'une gestion économique de l'activité séparée de la vie domestique, sont propices à la revendication des femmes pour la reconnaissance de leur travail par l'obtention d'un statut professionnel distinct de leur situation matrimoniale. Mais de la loi à son application, le chemin peut être long.

\section{La mise en cause du statut d'aide familial}

11 La contestation des femmes travaillant en agriculture ne parvient pas à s'exprimer directement, de manière frontale. Elle surgit à la suite d'importantes transformations de l'activité agricole qui, peu à peu, rendent légitimes leurs exigences de reconnaissance professionnelle.

12 Un gros effort de vulgarisation a lieu pour faire de l'agriculture une activité rentable selon des critères importés du monde industriel. Pour cela, les conseillers agricoles se réfèrent à l'ouvrage de référence conçu par des chercheurs de l'INRA se situant à la charnière d'une agriculture fondée sur une logique familiale de production et d'une activité obéissant aux critères de l'économie marchande (Chombart de Lauwe et al., 1969). Désormais, le travail agricole devient un « facteur de production » et non plus seulement 
l'expression de la solidarité familiale. L'évaluation monétaire des biens selon un prix de marché est évoquée face à leur définition patrimoniale et le travail acquiert une dimension temporelle pour en établir l'équivalent monétaire. Alors, apparaissent les catégories de travail et de non-travail jusque-là absentes en agriculture. Là, se trouve l'acte fondateur de changements dans une activité où depuis toujours la famille définit les règles de l'échange. Avant l'utilisation des méthodes de comptabilité de gestion, il était inconcevable de distinguer le travail «productif» du travail domestique. L'un et l'autre imbriqués dans le même espace et dans un temps unique, donnaient lieu à une seule et même trésorerie.

13 La référence au salariat utilisée par les économistes ruraux comme le moyen de moderniser l'activité agricole implique la césure entre l'univers domestique et l'univers professionnel. Dans ce contexte, les femmes qui travaillent en agriculture se trouvent doublement infériorisées : en appartenant à un secteur d'activité où le travail productif lui-même n'apparaît pas clairement, et puis en étant d'emblée affectées à la vie domestique comme lieu de consommation et non comme lieu de production.

En agriculture, le besoin de reconnaissance professionnelle ne peut rencontrer d'écho tant qu'il s'exprime au nom des femmes. Leur désignation sociale depuis la famille affaiblit la portée de leur revendication. En revanche, la contestation devient efficace dans la mesure où elle ne concerne pas seulement les femmes mais les jeunes et notamment les fils d'agriculteurs qui, participant à la production en tant qu'aide familial, se destinent à succéder à leurs parents. Ils ont pouvoir d'interpeller la logique familiale en tant que négation de leur travail. Les autres membres de la fratrie, partis pour faire des études, bénéficient de l'effort de celui qui, resté auprès de ses parents, fait fructifier le patrimoine familial sans en recevoir la reconnaissance financière si ce n'est sous forme "d'argent de poche». Ainsi, les fils et les filles travaillant dans la ferme familiale n'utilisent pas la même stratégie pour s'opposer à leur condition.

\section{Les hommes contestent la famille}

Pour exiger une reconnaissance de leur fonction de producteur, les jeunes agriculteurs mettent en cause directement la famille en la dénonçant comme la source de l'exploitation de leur travail contrairement à l'idéologie qui la fonde : lieu d'entraide et de solidarité. Michel Debatisse, jeune agriculteur du centre de la France devenu plus tard un des grands leader de la paysannerie française remet en cause la structure même de la production agricole en dénonçant l'exploitation familiale comme «l'exploitation de la famille» (Debatisse, 1964). En 1972, au Centre national des jeunes agriculteurs (CNJA) au congrès de Vichy, éclate le rejet du statut d'aide familial qui, établi selon les normes de la famille, s'oppose à tout principe de rémunération du travail de ses membres. Les jeunes se décrivent comme "travaillant en contrepartie du couvert, de l'habillement, du logement » et recevant « un peu d'argent de poche selon le bon vouloir de ses parents ». Ils crient leur colère dans les journaux : «Fini le temps des marginaux qui travaillent pour de l'argent de poche! fini le temps des spoliations légalisées au moment des partages! fini le temps des manceuvres sans responsabilité! Bref, il est fini le temps des aides familiaux! $»^{2}$

Se référant au salariat, les jeunes agriculteurs réclament une rémunération de leur travail ainsi que du pouvoir et des responsabilités dans les décisions de production en prévision de leur futur rôle de chef d'exploitation. La réponse juridique est le statut «d'associé d'exploitation » créé en 1973 pour se substituer à celui d'aide familial introduisant ainsi 
une reconnaissance du travail interne aux relations familiales notamment entre les générations et dans les faits, entre père et fils. Très peu de parents (à peine 300) consentent un tel contrat avec leurs enfants. Dans l'ensemble, ils s'érigent contre l'ingérence de l'État dans la famille. Au moment des débats parlementaires, certains députés manifestent leur désapprobation: "Nous avons nos enfants par nature et non par convention!» Les parents se montrent réticents à rémunérer leurs enfants sous la forme d'un «intéressement aux résultats de l'exploitation » car cela implique la participation directe de ces derniers à la gestion de l'entreprise paternelle. Ils répugnent à reconnaître un "congé-formation" pour que leur fils puisse acquérir de nouvelles techniques de production en dehors de l'influence paternelle. Un tel statut, même peu appliqué, est resté chargé de valeur symbolique, ouvrant la voie à la formation de sociétés de production entre parents et enfants, ces derniers exerçant leur activité en tant que chefs d'exploitation. Les GAEC (Groupement agricole d'exploitation en commun) entre père et fils se développent à partir des années 1970 en réponse à la contestation des aides familiaux masculins. Mais tout cela ne concerne pas les femmes. L'interdit de la loi empêchant deux époux d'être seuls associés (Barthez, 2000) maintient, sans le dire, l'épouse comme aide familiale de son mari contrairement à son fils qui obtient désormais la possibilité de bénéficier du même statut que son père. Bien sûr, la fille peut légalement former une société avec son père mais dans les faits, une telle situation n'a pas lieu car elle se heurte au-delà de l'agriculture, à la distinction entre famille et profession et à la division sexuelle correspondante affectant la femme en priorité au foyer.

\section{Les femmes fuient l'agriculture et refusent d'épouser un agriculteur}

Pour faire aboutir leurs exigences, les jeunes femmes ne disposent pas des mêmes moyens que les hommes puisqu'elles ne sont pas appelées, comme eux, à devenir chef d'exploitation. Leur trajectoire implique leur mariage avec un statut d'aide familial auprès de leur mari. Leur désir de quitter l'agriculture, s'alimente aussi de leur refus d'une vie domestique où elles se trouvent dépourvues d'initiative et de confort tant les dépenses pour la modernisation de l'exploitation sont prioritaires. Pour s'opposer à la place qui leur est faite, les femmes interviennent précisément là où elles sont prioritairement attendues, la vie de famille. Refuser d'épouser un agriculteur, quitter le milieu agricole forment ensemble leur manière spécifique de revendiquer une autre place dans l'agriculture. L'analyse statistique de ce mouvement révèle non seulement l'accroissement $\mathrm{du}$ célibat des hommes mais surtout des choix matrimoniaux très différents selon le sexe : les paysans se marient surtout avec des filles de paysans tandis que les filles de paysans épousent surtout des non-paysans: "Sur 100 agriculteurs qui se sont mariés en 1969, 58 ont épousé une fille de la même catégorie professionnelle tandis que sur 100 filles d'agriculteurs qui se sont mariées en 1969, 21 seulement ont épousé un agriculteur ou un salarié agricole. » (Jégouzo, 1972) De même, la raréfaction des filles aides familiales se fait sentir avec plus d'acuité que celle des fils de même statut : en 1970, parmi les descendants aides familiaux du chef d'exploitation, les femmes sont moitié moins nombreuses que les hommes. En effet, le Recensement général de l'agriculture (RGA) compte 368000 descendants aides familiaux masculins contre 179000 femmes descendantes de la même catégorie. 

atteinte aux normes culturelles de la paysannerie autour de l'alliance, de la transmission du patrimoine et de la division du travail. En s'opposant au mariage avec un agriculteur, les femmes invalident l'une des stratégies les plus importantes dans le milieu visant à réunir dans un même élan, la perpétuation de la lignée et la consolidation du patrimoine. Les recherches ethnographiques de cette époque soulignent les particularités de cet exode féminin qui n'est pas lié uniquement aux ressources économiques de la famille paysanne. Dans le sud de la France, Bourdieu montre combien la stratégie matrimoniale utilisée par les familles pour éviter la dispersion du patrimoine se trouve battue en brèche par l'exode féminin et le célibat masculin qui en résulte: «Chez les filles, on n'observe aucune relation significative entre l'émigration et la taille de la propriété ou le rang de naissance, les filles de bonne famille quittant même la terre dans une proportion légèrement plus élevée que les autres. " (Bourdieu, 1990)

19

En quittant le milieu agricole, les femmes ont touché l'un des aspects fondamentaux de l'organisation sociale de la paysannerie. Leur absence du monde agricole a paradoxalement révélé leur importance pour l'évolution de ce milieu. De là, celles qui sont restées ont pu exprimer directement leurs exigences pour accepter de vivre dans l'agriculture.

\section{Devenir agricultrice}

\section{Le refus du statut de femme au foyer : la vie domestique comme contrainte}

Les femmes, qui revendiquent un statut professionnel dans l'agriculture, doivent affronter un obstacle a priori, imprévisible : la modernisation du travail agricole qui, en allégeant leur tâche, est considérée comme un signe de progrès social tend cependant à les exclure de l'activité. Avec la mécanisation du travail, les hommes se sont emparés des tâches qu'effectuaient traditionnellement les femmes leur donnant ainsi la possibilité de se limiter à leur rôle de "femme au foyer» en référence à la condition de «la bourgeoise » (Martin-Fugier, 1983). Mais le contexte n'est plus à la valorisation exclusive de la vie domestique. Les femmes visent leur indépendance économique, le salariat étant le mieux adapté à cet objectif. La femme au foyer n'est plus enviable tandis que celle qui parvient à l'autonomie financière malgré ses attaches familiales correspond mieux au modèle féminin recherché.

21 La norme de l'assignation des femmes à la vie domestique comme leur véritable place fait obstacle à celles qui cherchent à travailler en agriculture comme une profession leur permettant de répondre à leurs besoins d'indépendance économique. Alors que pour les hommes l'évolution technologique se présente comme le moyen de réduire leur effort tout en augmentant la productivité de leur travail, pour les femmes, elle signifie un danger : l'accroissement de leur dépendance. Si l'on considère le couple comme unité sociale, l'homme au travail, la femme à la maison, sont les termes de la complémentarité objective entre les époux. Si l'on considère l'individu, «la femme au foyer » se trouve dépendante de l'activité de production exercée par son mari en dehors d'elle. Elle n'accède plus ni à l'information, ni aux responsabilités concernant une profession qui pourtant se déroule sur les lieux mêmes de la vie domestique. En agriculture, les femmes ressentent une telle transformation comme une éviction.

Économie rurale, 289-290 | Septembre-décembre 2005 

réalisées jusque-là à l'aide de la traction animale, modifie profondément la place de l'agriculture parmi les autres secteurs d'activité (Mendras, 1967). Traditionnellement considérée comme un état et non comme un métier, l'agriculture, en se modernisant utilise les moyens de l'entreprise industrielle et en cela emprunte ses caractéristiques notamment les techniques de gestion d'entreprise. Les femmes qui souhaitent participer à l'activité agricole adoptent cette logique économique du travail. En s'exprimant en termes « d'emploi » pour elles (Péroche, 1975) dans l'exploitation agricole, elles se placent délibérément en dehors du rôle qui leur est attribué dans la famille et trouvent dans cette logique économique les raisons d'une émancipation.

L'agriculture étant structurée comme production familiale, il est difficile de réprouver à la fois la situation d'aide familial tout en revendiquant de travailler dans l'exploitation agricole tandis que la modernisation de l'activité libère les femmes des tâches qu'elles avaient en charge. Les femmes doivent dire à la fois qu'elles ne veulent plus être ni aide familiale ni femme au foyer mais qu'elles revendiquent un travail sur la ferme assimilable à un emploi qui leur soit reconnu à titre personnel. Or, l'exploitation familiale est précisément celle dont le mari est le chef. Alors, apparait une nouvelle contradiction: comment peuvent-elles obtenir une identité professionnelle dans un système placé sous l'autorité de leur mari?

\section{2. Évolution de la famille et revendications des femmes en agriculture}

On ne peut expliquer l'évolution du statut des femmes en agriculture sans évoquer simultanément celle de la famille elle-même. Dans la mesure où celle-ci est le cadre de l'activité agricole, les changements liés aux relations entre époux retentissent sur la place reconnue à la femme dans l'exploitation agricole.

Le développement des mouvements féministes à partir des années 1960 ouvre un espace de revendications pour une meilleure reconnaissance du travail des femmes et un accroissement de leur autorité tant dans la famille que dans le monde du travail. En 1967, la reconnaissance légale de la contraception, puis celle de l'avortement en 1975 autorisent la femme à gérer sa procréation. Avec la réforme des régimes matrimoniaux de 1965, le mari perd le droit de s'opposer à la volonté de son épouse d'exercer une activité professionnelle. Il perd aussi le pouvoir exclusif de gérer les biens acquis en commun tandis que son épouse récupère la totale gestion de ses biens propres. En 1970, le remplacement de la notion de "chef de famille» par celle "d'autorité parentale» reconnaît à l'épouse une autorité sur les enfants, à partager avec son mari. Enfin, avec la montée des divorces en France, la loi sur « le divorce par consentement mutuel » en 1975, allège la procédure de séparation des couples.

Dans ce contexte de dix années de changements profonds de la famille, l'épouse d'agriculteur s'autorise peu à peu à revendiquer une place dans l'exploitation agricole autre que celle d'aide familial : «Travailler bénévolement sur l'exploitation, en bouche-trou en personne en surnombre qui s'y occupe, cela nous ne l'acceptons plus. Ce n'est pas un salaire que nous réclamons, non, mais nous voulons sentir que notre travail est efficace et reconnu comme tel dans l'exploitation, la famille, la société. Si nous sommes servantes de tout le monde, dans la famille, cela ne va pas. $»^{3}$ 
27 Les journaux agricoles se remplissent de témoignages de femmes développant leur exigence d'identité professionnelle dans la ferme familiale où elles travaillent. Par exemple, des femmes du sud de la France revendiquent «la responsabilité entière d'un atelier de $\mathrm{A}$ à $\mathrm{Z}$ et un revenu à soi ${ }^{4}$. De même, elles demandent qu'il soit possible de constituer des ateliers d'élevage sous «l'entière responsabilité des femmes » y compris la commercialisation des produits, en dehors de toute tutelle du mari ${ }^{5}$. Se peut-il d'en passer par l'accord du mari pour obtenir une place dans la ferme dont l'épouse en revendique l'exclusive responsabilité ? Sans le dire, les femmes dévoilent la réalité de relations de domination entre époux au lieu de la complémentarité proclamée. En se désignant " agricultrices », elles dénoncent l'organisation d'une production fondée sur des unités familiales avec un chef unique. Aussi prennent-elles à leur profit l'entrée des femmes dans le monde du travail industriel pour légitimer leur besoin d'identité professionnelle. En s'érigeant contre la volonté qu'elles décrivent « masculine » de « libérer la femme des travaux agricoles", elles affirment combien "c'est une fausse piste de vouloir l'agriculture sans nous (les femmes), la profession agricole peut au contraire offrir aux femmes des postes variés, intéressants, qui correspondent parfaitement à leurs possibilités. Et ces postes sont aussi valorisants que ceux que nous pourrions obtenir dans le commerce et l'industrie. ${ }^{6}$

La commission féminine du CNJA s'érige contre les formations destinées aux filles dans les écoles ménagères (Caniou, 1980) car elles les confinent dans l'apprentissage des tâches qui ne concernent que l'univers domestique au lieu de les préparer à "prendre des responsabilités dans l'exploitation ». Aussi revendiquent-elles l'acquisition de " compétences techniques mais aussi des données au niveau juridique et comptable » Dans ce mouvement de désigner leur travail comme une profession à acquérir en agriculture, les femmes créent peu à peu la consistance d'une fonction autre que celle directement liée à leur mariage.

Les femmes du CNJA expriment clairement ce qui apparaît comme un dilemme pour elles : devenir agricultrice ou bien épouser un agriculteur. «La mécanisation de l'agriculture ayant rendu les travaux moins pénibles, de plus en plus aujourd'hui, on trouve des femmes célibataires qui choisissent d'être agricultrice. Si on les reconnaît comme telles, il n'en est pas de même lorsqu'elles sont mariées avec un agriculteur : elles deviennent femmes d'exploitant. $»^{8}$ Elles montrent ainsi combien la question de leur reconnaissance professionnelle se trouve inscrite dans l'organisation structurelle de l'activité et non pas seulement liée au bon vouloir ponctuel d'un groupe d'hommes. Mais la volonté politique de maintenir le groupe domestique au fondement de l'unité productive s'oppose de fait à l'autonomie des agricultrices sauf à le devenir en se maintenant célibataires ou encore en cas de veuvage. Le mariage est évoqué comme un obstacle radical à la reconnaissance du travail des femmes. Ainsi l'expriment-t-elles en 1983, aux États généraux du développement agricole : "Si tu es une femme, le jour où tu épouses un agriculteur, tu n'es plus rien, tu n'as plus de droit, plus rien. Tu ne redeviens quelqu'un que lorsque, par malheur, ton bonhomme meurt.» (Barthez, 1984) A.-M. Crolais, agricultrice et responsable au CNJA écrit dans un livre qui fit grand bruit : "Il est inconcevable qu'aujourd'hui, une femme qui travaille plus de six heures par jour sur une exploitation agricole, participant aux travaux, mais aussi à la gestion de celle-ci, responsable financièrement, comme son mari, du succès ou de l'échec de l'installation, soit... un zéro social. » (Crolais, 1982)9 Le couple - et non l'individu - comme unité de base, est manifeste au moment de la création du GAEC, forme sociétaire en agriculture permettant une meilleure productivité par regroupement d'unités petites ou moyennes. En fait, à l'origine, il s'agit de regrouper 
des unités familiales et non des individus (Barthez, 2000). Créé en 1962, le GAEC est établi comme une société civile de personnes permettant à plusieurs agriculteurs de se réunir en excluant le cas où deux époux en seraient les seuls associés. Pour que mari et femme soient partenaires de la même la société, il faut que l'effectif des associés compte au moins un membre de plus. Par exemple, l'épouse peut devenir membre de GAEC, celui-ci étant déjà constitué entre le père et le fils. Une telle restriction s'explique par la volonté politique exprimée dans la loi d'Orientation agricole de 1960 de moderniser l'agriculture tout en la conservant sous une forme familiale de production. Le refus de considérer les épouses comme agricultrices séparément de leur mari, se réfère au couple et non à l'individu comme unité de base de la famille et de l'unité de production.

\section{Épouse d'agriculteur active}

31 À la suite de la période de célibat des agriculteurs lié à l'exode féminin, apparaît un nouveau phénomène : la formation de couples dont l'épouse travaille hors de la ferme, le plus souvent comme salariée.

\section{Femmes actives hors de la ferme familiale : leur fonction critique}

Face aux témoignages de femmes qui se disent agricultrices, la presse agricole enregistre les propos de femmes mariées à des agriculteurs, déjà professionnalisées hors de l'agriculture avant leur mariage et qui entendent conserver leur emploi. Les conjoints s'expriment également et disent comment ils ont adapté leur système de culture en l'absence de leur épouse sur l'exploitation. Selon ces nouveaux couples, l'exercice de l'agriculture est compatible avec le travail de la femme hors de la ferme familiale, et le mariage d'un agriculteur avec une femme dotée de reconnaissance professionnelle est possible. Des institutrices, des assistantes sociales, des infirmières témoignent dans la presse agricole d'une telle union. Elles affirment leur attachement à leur profession distincte de celle de leur mari non seulement par goût de leur métier mais aussi par rejet de la condition faite à la paysanne "Quand je me suis mariée, j'avais mon métier (infirmière) et je ne voulais pas le quitter pour devenir agricultrice, la vision que j'avais des agricultrices dans la région (les Vosges) n'était pas réjouissante et leur vie ne me semblait pas très épanouissante. Une agricultrice était la bonne de son mari et la bonne à tout faire de l'exploitation. C'était l'esclavage. $\aleph^{10}$ De son côté, le mari affirme son choix délibéré pour une femme qui n'appartient pas au même milieu professionnel que lui, même s'il a dû adapter son système de production du fait de l'absence de son épouse sur l'exploitation : «J'ai toujours souhaité ne pas avoir une femme agricultrice. Pourquoi un agriculteur serait-il obligé d'épouser une fille d'agriculteur? Le facteur ne doit pas épouser la postière et l'instituteur, une institutrice. Alors, pourquoi en serait-il autrement en agriculture? Déjà à 17 ans, je ne pouvais admettre cela et j'ai autant fréquenté des jeunes filles d'autres milieux que d'agricultrices. »

33 Ces propos qui expriment la transgression de normes culturelles pourtant bien établies en agriculture à travers les relations patrimoniales (Bourdieu, 1972) annoncent une ouverture des alliances, et ce faisant, cassent l'organisation traditionnelle du ménage paysan. Des enquêtes (Derieux, 1975) révèlent la vision qu'ont les épouses actives hors de l'exploitation, de la condition de la femme en agriculture : «Elles regrettent la confusion presque inévitable lors de l'accomplissement des tâches agricoles, entre vie familiale et vie professionnelle... De plus, le travail de la femme sur la ferme est trop facilement considéré comme 
"naturel", découlant de sa situation même d'épouse d'agriculteur. Sa contribution à la marche de l'exploitation lui paraît alors dépréciée, bien plus, souvent inutile. "

Le regard de ces femmes sur l'activité agricole ouvre l'espace de la contestation pour celles qui cherchent à améliorer leur sort en travaillant dans la ferme familiale. L'avènement de cette nouvelle catégorie d'épouses forme une référence pour celles qui veulent devenir agricultrice. Elles trouvent là une légitimité pour exiger plus de responsabilité et plus d'autonomie dans leur travail de production. Il est curieux de voir à travers la presse les témoignages des unes et des autres apparemment sans liens et pourtant complémentaires comme s'ils se répondaient en écho sur le thème de l'identité professionnelle de l'épouse d'exploitant : pour les unes, il s'agit de privilégier le travail à l'extérieur pour éviter de basculer dans la situation dévalorisante de l'aide familiale tandis que pour les autres, il faut se battre pour acquérir un statut d'agricultrice qui les sépare de la condition de l'aide familiale corvéable et travailleuse de l'ombre. Les femmes participant aux mouvements féministes s'opposent à la distinction faite en économie entre le travail domestique et le travail productif. Pour elles, dans les sociétés industrielles, toute activité est susceptible d'évaluation monétaire. Pour la première fois, dans les années 1980, une estimation statistique du travail domestique en termes du travail productif est osée, révélant une contribution féminine à la richesse nationale supérieure à celle des hommes. (Chadeau et Fouquet, 1981). Une telle approche réalisée au sein de l'Institut national de la statistique et des études économiques (INSEE) par des cadres féminins, enfreint la norme la plus instituée du travail, et retentit dans les médias ${ }^{11}$ avec la violence d'une transgression.

La proportion des épouses d'agriculteurs exerçant une activité non agricole n'a cessé d'augmenter. De $7 \%$ en 1970, elle passe à $40 \%$ en 2000. Parmi les jeunes ménages, les deux tiers des épouses sont dans ce cas. Toutefois, cette croissance spectaculaire ne saurait être interprétée uniquement comme la conquête de l'indépendance féminine dans les ménages agricoles. Le salaire rapportée par l'épouse intervient aussi dans la stratégie économique du couple pour le maintien de l'exploitation familiale (Bessières, 2004).

\section{L'épouse agricultrice ou l'agriculture « métier de couple »}

Dans leurs revendications, les femmes ne remettent pas en cause l'activité agricole fondée sur la famille, ses relations, et sa logique patrimoniale. Ainsi, elles ratifient l'appartenance de l'agriculture au secteur des Indépendants où l'installation est avant tout "une affaire de couple» et non une démarche individuelle. Que l'épouse aide directement son mari ou qu'elle se consacre à la vie domestique ou encore qu'elle soit salariée à l'extérieur, l'entreprise familiale mobilise les deux conjoints sans quoi elle ne pourrait exister (Bertaux-Wiame, 2004). Le syndicalisme agricole massivement masculin consent à endosser le dossier des agricultrices dans la mesure où celles-ci s'expriment en tant qu'épouses d'agriculteur et non en tant qu'individus (Lagrave, 1987).

La commission féminine du syndicat agricole le plus représenté ${ }^{12}$, énonce l'avènement de l'agricultrice en se référant au couple comme cadre de l'identité professionnelle: " L'agriculture, c'est un métier de couple, c'est tantôt l'un, tantôt l'autre, souvent les deux ensemble qui prennent telle ou telle décision, qui orientent l'exploitation... c'est donc plus qu'un statut d'agricultrice qu'il faut mettre en place... c'est un véritable statut spécifique aux époux qui exploitent ensemble une exploitation agricole. $»^{13} \mathrm{Au}$ lieu du salariat qui individualise, les femmes optent pour une reconnaissance professionnelle depuis la conjugalité familiale : 
"Nous voulons une promotion des personnes par une vie de couple, nous voulons nous épanouir en vivant à deux, en travaillant à deux, en gérant à deux une exploitation, un patrimoine. Pour nous, c'est une valeur fondamentale. $»^{14} \mathrm{En}$ réalité, les femmes ne remettent nullement en cause la structure familiale de production comme la source de leur absence de statut professionnel. Au contraire, elles se montrent très attachées à une agriculture familiale et, dans ce contexte, demandent un nouveau partage. Le couple est la référence d'où elles revendiquent des rapports égalitaires au lieu de la distinction hiérarchisée faisant le mari chef d'exploitation et son épouse, aide familiale.

Le statut de l'agricultrice «co-exploitante » résultant de la loi d'Orientation agricole de $1980^{15}$ correspond à une reconnaissance de l'activité de l'épouse d'exploitant. Dans cette logique, l'épouse acquiert le droit "d'accomplir les actes d'administration concernant les besoins de l'exploitation » et le mari, de seul chef responsable qu'il était détient désormais " une capacité de gestion à partager avec son épouse : lorsque des époux exploitant ensemble et pour leur compte un même fonds agricole, ils sont présumés s'être donnés mandat d'accomplir les actes d'administration concernant les besoins de l'exploitation. » Il s'agit ici, de reconnaître une égalité de droits entre les époux concernant le travail et les responsabilités professionnelles. Désormais l'épouse, autant que le mari, détient le droit de représenter l'exploitation dans les différentes instances syndicales et professionnelles. Mais il est clair qu'il s'agit d'une identité professionnelle à partager avec le mari, depuis l'existence du couple, et non d'un droit personnel (Barthez, 1984). Tout dépend de l'entente au sein du couple pour que l'épouse puisse devenir réellement co-exploitante au-delà de son statut traditionnel d'aide familiale. Toutefois depuis cette loi, la première en faveur de l'activité des femmes en agriculture, les épouses modifient peu à peu leur comportement dans la désignation d'elles-mêmes. Elles tendent à se déclarer au grès des recensements «agricultrice à titre principal» et non plus «aide familiale». Dans ce contexte la loi s'exerce beaucoup plus comme une incitation au changement culturel que comme pure fonction normative (Assier-Andrieu, 1996). Enfin, la dernière loi d'Orientation agricole ${ }^{16}$ instituant «le conjoint collaborateur" marque un progrès dans la reconnaissance professionnelle du travail féminin et la protection sociale de l'agricultrice. Avec plus de responsabilité, plus de visibilité de son travail, l'épouse "collaboratrice» tend, plus qu'autrefois à se déclarer active "à plein-temps » s'affirmant ainsi détentrice d'une activité professionnelle y compris dans l'exploitation familiale. En même temps que s'affirme son statut, se précise son activité.

\section{L'épouse chef d'exploitation, coassociée de son mari}

En agriculture, la création d'entreprise et la transmission sont très liées à la reproduction familiale et dans cette logique, la fonction de chef d'exploitation revient au fils et non à la fille. Les femmes qui deviennent chef d'exploitation le sont par défaut. En cas de veuvage, l'épouse assure la continuité, ou bien quand le mari part à la retraite, il transmet l'entreprise à son épouse qui, étant plus jeune, accumule grâce à ces quelques années précédant son départ, de meilleures conditions de retraite pour elle-même. Ainsi a-t-on coutume de constater l'âge avancé des femmes chef d'exploitation (50 ans et plus) ainsi que leur faible proportion (10 à $15 \%$ ). Mais l'évolution la plus saisissante est celle du développement des sociétés, particulièrement favorable aux femmes. Entre 1988 et 2000, leur nombre a presque doublé passant de 61000 à 117 000. Et dans l'intervalle, l'effectif des femmes chefs d'exploitation ou membres de sociétés s'est accru de $13 \%$ tandis que 
celui des hommes a diminué de $37 \%$. En 1988, elles représentaient $15 \%$ de l'ensemble, en 2000 , elles atteignent $24 \%$ (Rattin, 2002).

La multiplication des sociétés en agriculture est liée à l'apparition en 1985 d'une nouvelle forme juridique autorisant (contrairement au GAEC) les époux à être les seuls associés : l'Entreprise agricole à responsabilité limitée (EARL) ${ }^{17}$ Cette nouvelle formule sociétaire ouvre un espace d'identité $a$ priori insoupçonné pour les épouses dans la mesure où cette loi visait d'abord à limiter la responsabilité financière des associés en cas de faillite de l'entreprise. En étant juridiquement des associés et pas seulement des époux, les conjoints peuvent organiser leurs relations selon une autre structure que la seule alliance matrimoniale pour individualiser leurs tâches et leurs responsabilités professionnelles. Là est la version la plus moderne de l'agriculture «métier de couple » au plus près du modèle de la famille contemporaine individualisant chacun de ses membres (de Singly, 1997). En 1988, les EARL ne représentaient que 2,3\% de l'ensemble des sociétés tandis qu'aujourd'hui elles atteignent les $46 \%$.

41 L'évolution des femmes en agriculture depuis l'aide familiale jusqu'à la fonction de chef d'exploitation présente des caractères comparables à celle des femmes appartenant aux autres catégories d'indépendants (Fouquet, 2005). On assiste d'abord à l'effondrement de l'effectif des aides familiales : pour le secteur agricole, entre 1988 et 2000, il passe de 530 000 à 213000 soit une baisse de $60 \%$. Les femmes chefs d'entreprise se trouvent à la tête de petites unités, se consacrent de préférence aux élevages «hors sol » d'ovins et de caprins, ou à la viticulture. Elles dirigent rarement des exploitations de grandes cultures ou des élevages bovins. Par ailleurs, on sait que les femmes du secteur Indépendant travaillent beaucoup plus fréquemment que les hommes dans les services: $61 \%$ des femmes, $41 \%$ des hommes (Fouquet, 2005). En agriculture, leur contribution au développement des activités tertiaires est essentielle. L'accueil à la ferme, la transformation et la vente directe des produits fermiers sont assurés par les agricultrices (Giraud, 2001). Aujourd'hui, la presse agricole rapporte les nouveaux comportements des femmes qui les placent directement au contact des consommateurs. Sans être chefs d'exploitation en titre, elles deviennent des acteurs économiques à part entière à partir de productions autrefois limitées à la consommation familiale ${ }^{18}$. Elles découvrent de nouvelles filières de transformation et de vente (huile de colza) à partir de productions déjà pratiquées sur la ferme ${ }^{19}$. Elles sont également à l'initiative de marchés de proximité où « les consommateurs sont assurés de rencontrer exclusivement des producteurs du pays». En lien avec le milieu local, les agricultrices entrent de plain-pied dans une définition de l'agriculture qui cherche à s'inscrire dans la ruralité (Lagrave, 1999).

\section{Conclusion}

Les femmes ayant acquis une reconnaissance juridique de leur travail ont une histoire qui s'enracine dans la condition de la femme au foyer, aide familiale, et en charge du travail domestique. Tandis que les hommes ont une appartenance continue à l'univers professionnel, celle des femmes est récente. Leur accès au monde du travail interroge sur l'exercice et la définition sociale de professions jusque-là occupées par des hommes ou encore de celles, plus novatrices, utilisant les compétences mises en œuvre dans la vie domestique (Battagliola, 2004). En agriculture, comme dans le petit commerce ou l'artisanat, les femmes accèdent parfois à la tête des entreprises, mais elles y sont rarement seules. Elles y figurent aux côtés de leur mari, tenant un rôle qui, bien 
qu'essentiel au sein de l'activité, est passé sous silence ou considéré comme secondaire (Zalc, 2005). L'ancrage familial de l'activité reste un des éléments de la définition professionnelle. Il n'existe pas de frontière nette qui distingue l'entreprise de la famille. Appartenant aux deux, les agricultrices sont à même d'établir des liens entre une activité de production et son utilisation consommatrice dont elles connaissent les aspects depuis leur affectation à la vie domestique. Il reste à explorer en quoi leur histoire singulière nourrit-elle leur action sur la scène professionnelle, lieu traditionnellement masculin?

Les femmes parvenant à « percer » sur le terrain de la profession se voient souvent dans l'obligation "d'être deux fois plus compétentes", leur référence première étant le comportement masculin. Peut-on dire aujourd'hui, qu'elles ont acquis une assise suffisante pour ne plus s'imposer d'atteindre les performances masculines mais plutôt pour s'affirmer et reconnaître leur savoir-faire spécifique? Parviennent-elles à développer leur pratique dans la sphère professionnelle en référence à leur savoir généalogique acquis au fil de leur inscription dans l'univers de la consommation et du «hors travail» sans pour autant se sentir traversées par la dévalorisation liée à la « féminisation » des métiers?

Que les femmes puisent dans leur histoire d'appartenance à un autre univers social que le monde du travail forme aujourd'hui une ressource plus appropriée que l'imitation du savoir-faire masculin. L'étude des rapports de genre est délivrée de son caractère tabou. Les sciences sociales montrent combien la vie domestique et le monde du travail interfèrent très au-delà du cas particulier de l'agriculture, la parenté élective se substituant parfois à la parenté biologique (Weber et al., 2003). Si l'on admet que le comportement masculin n'est pas unique et n'a plus valeur de modèle, un besoin de recherche ethnographique se fait sentir pour saisir comment les femmes interviennent dans le monde du travail lorsqu'elles ont en charge des responsabilités collectives, politiques, comment s'exprime leur capacité de négociation dans l'univers marchand de relations, comment elles interviennent dans les rapports de concurrence et les conflits d'intérêts, et enfin comment se concrétisent les finalités de leur action à l'intersection de la vie professionnelle et de la vie familiale. L'agriculture reste exemplaire de l'interférence entre ces deux aspects de la vie sociale.

\section{BIBLIOGRAPHIE}

Agreste Cahiers. Recensement agricole 2000. Décembre 2001, n 3-4.

Allauzen M. La paysanne française aujourd'hui. Gonthier, Paris 1967.

Assier-Andrieu L. Le droit dans les sociétés humaines. Nathan, Paris, 1996, 322 p.

Barthez A. Famille, travail et agriculture. Economica, Paris, 1982, 193 p.

Barthez A. Le droit comme expression culturelle. Processus de légalisation du travail familial en

agriculture : le cas du GAEC. Revue de Droit Rural, Poitiers, 2000, n² 288, p. 621-632.

Barthez A. Femmes dans l'agriculture et travail familial. Sociologie du travail, 1984, $\mathrm{n}^{\circ} 3$. 
Battagliola F. Histoire du travail des femmes. La Découverte, Repères, Paris, 2004, 124 p.

Bécouarn M.-C. L'évolution du travail des femmes d'agriculteurs dans les exploitations d'élevage des Côtes $d u$ Nord. Thèse de Sociologie, Université Paris I, 1970, 322 p.

Bertaux-Wiame I. Devenir indépendant, une affaire de couple. L'Harmattan, Cahiers du Genre, Paris, 2004, $\mathrm{n}^{\circ} 37$, p. 13-41.

Bessière C. « Vaut mieux qu'elle travaille à l'extérieur ! "Enjeux du travail salarié des femmes d'agriculteurs dans les exploitations familiales. L'Harmattan, Cahiers du Genre, Paris, 2004, nº 37, p. 93-115.

Bourdieu p. Les stratégies matrimoniales dans le système de reproduction. Annales, Paris, $1972, \mathrm{n}^{\circ} 4-5$, p. 1105-1127.

Bourdieu p. Reproduction interdite. La dimension symbolique de la domination économique, Études Rurales, 1990, $\mathrm{n}^{\circ}$ 113-114. Cet article fait référence à une recherche du même auteur réalisée dans les années 1960, «Célibat et condition paysanne », Études Rurales, 1962, n 5-6.

Caniou J. L'enseignement agricole féminin de la fin du XIX ${ }^{e}$ siècle à nos jours. Thèse de sociologie, Université Paris V, 1980, 316 p.

Chadeau A., Fouquet A. Peut-on mesurer le travail domestique ? Économie et Statistique, 1981, n 136. Article faisant la synthèse de l'étude «L travail domestique : essai de quantification » parue dans Archives et Documents, 1981, $\mathrm{n}^{\circ} 32$.

Chombart de Lauwe J., Poitevin J., Tirel C. Nouvelle gestion des exploitations agricoles.

Crolais A.-M. L'agricultrice. Ramsay, Paris, 1982.

Daune-Richard A. M. Hommes et femmes devant le travail et l'emploi. In : Blöss T. (Ed.), « La dialectique des rapports hommes-femmes », PUF, Paris, 2002, p. 127-146.

Debatisse M. La révolution silencieuse. Le combat des paysans. Calmann-Lévy, Paris, 1964.

Derieux F. Les épouses d'agriculteurs exerçant une activité professionnelle non agricole. Sfer, Économie Rurale, $1975, \mathrm{n}^{\circ} 110$, p. 45-49.

Duby G. Histoire de la France Rurale. Tome III « Apogée et crise de la société paysanne de 1789 à 1914 », Seuil, Paris, 1976, p. 344.

Fouquet A. L'invention de l'inactivité. In Travail, Genre et Sociétés, 2004, n 11, p. 47-62.

Fouquet A. Les femmes chefs d'entreprise : le cas français. In Travail, Genre et Sociétés, 2005, nº 13, p. 31-51.

Giraud C. Chambres d'hôtes à la ferme et autonomie de la femme en agriculture. Thèse de sociologie, Université Paris V, Paris, 2001, $473 \mathrm{p}$

Gramain A., Weber F. Modéliser l'économie domestique. In Weber F. (Dir.), « Charges de famille : dépendance et parenté dans la France contemporaine », La Découverte, Paris, 2003, p. 9-42.

Jégouzo G. L'ampleur du célibat en agriculture. Économie et Statistique, 1972, n³4.

Kalaora B., Savoye A. Les inventeurs oubliés : Le Play et ses continuateurs aux origines des sciences sociales. Ed. Champ Vallon, Seyssel, 1989, 296 p.

Lagrave R.-M. (dir). Celles de la terre. Agricultrice : l'invention politique d'un métier. EHESS, Paris, 1987, $254 \mathrm{p}$.

Lagrave R.-M. Les agricultrices et la récurrence de l'économie du dévouement. Aménagement de la Nature, Paris, $1999, n^{\circ} 132$, p. 41-62. 
Le Play F. Les Mélouga. Une famille pyrénéenne au XIX siècle. La famille souche. Questions de méthode, Nathan, Essais et Recherches, Paris, 1994

Martin-Fugier A. La bourgeoise. Biblio-Essais, Paris, 1988.

Mendras H. La fin des paysans. Innovations et changements dans l'agriculture française. SEDEIS, Futuribles, Paris, 1967, 245 p.

Péroche J. À la recherche d'un emploi féminin sur l'exploitation : bilan, action, entreprise. ANDAFNCETA, Deux Sèvres, 1975, 55 p.

Rattin S. L'agriculture au féminin se professionnalise. Agreste Cahiers, 2002, $\mathrm{n}^{\circ} 2$.

Rattin S. La vie des femmes dans les exploitations. Agreste, Études, 1990, nº 5.

Schweitzer S. Les femmes ont toujours travaillé. Une histoire du travail des femmes au XIXe et XXe siècles. Odile Jacob, Paris, 2002, 324 p.

Ségalen M. Mari et femme dans la société paysanne. Flammarion, Paris, 1980, 150 p.

Sèverac $\mathrm{Cl}$. Travail et identité des femmes en agriculture, un exemple : le pays d'Apt en Vaucluse. Thèse de sociologie, Université d'Aix-Marseille, 1980, 302 p

Singly (de) F. Sociologie de la famille contemporaine. Nathan, Paris, 1997, 128 p.

Weber F., Gojard S., Gramain A. Charges de famille. Dépendance et parenté dans la France contemporaine. La Découverte, Paris, 2003.

Zalc C. Femmes, entreprises et dépendances. Les entrepreneuses étrangères à Paris dans l'entre-deuxguerres. In Travail, Genre et Sociétés, 2005, n 13, p. 51-74.

\section{NOTES}

1. In Allauzen M., page 46.

2. Jeunes Agriculteurs, "Un statut pour les aides familiaux : associé d'exploitation ", n 262, 1972.

3. $C f$. Entreprises Agricoles, janvier 1969.

4. In Agri Sept, $1977, \mathrm{n}^{\circ} 658$.

5. Cf. Entreprises Agricoles, mai, septembre 1974.

6. Cf. Entreprises Agricoles, octobre 1977.

7. CNJA, Aujourd'hui, des agricultrices, Rapport Simone Martin, journées d'études, Caen, 1975.

8. Ibid

9. In Crolais A.-M., page 200.

10. Un agriculteur vosgien: "Épouser une agricultrice n'est pas une obligation.» In Entreprises Agricoles, novembre 1975. Entretien avec un couple formé par un agriculteur et son épouse salariée.

11. «L'INSEE s'amuse. Vous savez, ce fabuleux et sévère institut national de la statistique et des études économiques, merci pour lui, où vous pouvez rencontrer un polytechnicien au centimètre carré... ", France Inter, le 2 avril 1981. In A. Fouquet, La valorisation du travail domestique. L'histoire d'une étude, $\mathrm{n}$ - 2321/413, 1982.

12. La FNSEA (Fédération nationale des syndicats d'exploitants agricoles).

13. In Entreprises Agricoles, octobre 1976.

14. In Entreprises Agricoles, Statut des épouses d'agriculteurs : où en est-on? décembre 1977.

15. Loi d'Orientation agricole, $\mathrm{n}^{\circ} 80-502$ du 4 juillet 1980.

16. Loi d'Orientation agricole, $\mathrm{n}^{\circ}$ 99-574 du 9 juillet 1999. 
17. Loi $n^{\circ}$ 85-697 du 11 juillet 1985 relative à l'Entreprise unipersonnelle à responsabilité limitée et à l'Exploitation agricole à responsabilité limitée.

18. «Des oies et des canards gras pour rentabiliser l'investissement", In La France Agricole, 2003, n - 2985.

19. L'information agricole, $2003, n^{\circ} 769$.

\section{RÉSUMÉS}

Devenir agricultrice n'est pas décrit ici comme l'accès des femmes à un univers professionnel distinct de la vie domestique mais plutôt comme un travail de la famille sur elle-même, capable d'intégrer la dimension marchande au sein des relations de parenté. Pour cela, sont utilisés outre les travaux de sociologues sur les rapports de genre, les apports du droit, et aussi les publications de la presse révélant les différentes stratégies des femmes pour faire reconnaître leur activité dans un univers qui reste familial.

Becoming a woman farmer is not described here as a way for women to enter a professional agricultural world distinct from the traditional household activities, but rather as a transformation effort of the family itself, able to integrate the business dimension within family relationships. To do so, in addition to sociologists works on the gender connexions, we have used legal contributions as well as press publications that point out the different strategies elaborated by women to make their professional agricultural activities admitted in a world that stays very family oriented.

\section{INDEX}

Mots-clés : agriculture, femme, profession, travail

Code JEL J12 - Marriage; Marital Dissolution; Family Structure

Keywords : farm family, gender, woman, work

\section{AUTEUR}

\section{ALICE BARTHEZ}

Institut national de la recherche agronomique, Dijon 